\title{
Bilirubin accumulation and Cyp mRNA expression in selected brain regions of jaundiced Gunn rat pups
}

Silvia Gazzin, Jaroslav Zelenka, Lucie Zdrahalova, Renata Konickova, Carlos Coda Zabetta, Pablo J. Giraudi, Andrea L. Berengeno, Alan Raseni, Maria C. Robert, Libor Vitek and Claudio Tiribelli

Pediatr Res 71:653-660 (2012); doi:10.1038/pr.2012.23

In this article, the affiliations listed for two authors appeared incorrectly.

The correct affiliations for Libor Vitek are:

Institute of Clinical Biochemistry and Laboratory Diagnostics, 1st Faculty of Medicine, Charles University, Prague, Czech Republic; and 4th Department of Internal Medicine, 1st Faculty of Medicine, Charles University, Prague, Czech Republic.

The correct affiliations for Claudio Tiribelli are:

Fondazione Italiana Fegato (Italian Liver Foundation), Trieste, Italy; and Department of Medical Sciences, Università degli Studi di Trieste, Italy.

The publisher regrets these errors. 